\title{
EL PROCESO DE DECISIÓN DE LAS VACACIONES FAMILIARES Y SU RELACIÓN CON LA SATISFACCIÓN DE LA PAREJA
}

The decision process of family holidays and its relationship with the satisfaction of the couple

\author{
MARÍA-MERCEDES ROJAS-DE-GRACIA ${ }^{1}$ \\ Universidad de Málaga, Andalucía Tech \\ PILAR ALARCÓN-URBISTONDO \\ Universidad de Málaga, Andalucía Tech
}

\begin{abstract}
Resumen: Aunque la satisfacción con el destino turístico es un tema que ha atraído el interés de muchos investigadores, no ha sido tan común para el caso de las vacaciones familiares. Además, en pocas ocasiones se han realizado trabajos que recojan las respuestas de hombres y mujeres por separado, lo que permite un análisis más exhaustivo de las mismas. En este trabajo, se ha analizado una muestra de 375 parejas, cuyos miembros contestaron a sendos cuestionarios por separado, tratándose de un tamaño de muestra considerable en estudios familiares. Se concluye que, en general, cuando las familias viajan, la satisfacción con el destino suele ser alta, sin diferencias significativas entre sexos, aunque siendo ligeramente mayor en el caso de las mujeres. La aparición de conflicto entre la pareja durante el proceso de elección del destino y el hecho de viajar a un destino nacional o internacional son las variables más influyentes en la satisfacción, tanto para hombres como para mujeres. Las implicaciones prácticas indican que los gestores de los destinos turísticos deben tener muy presentes a los turistas nacionales en sus estrategias, y no deben dejar de lado el uso de argumentos racionales en sus campañas de comunicación que permitan argumentar la conveniencia del destino en caso de aparición de conflicto entre la pareja.
\end{abstract}

Palabras clave: Satisfacción con el destino turístico, vacaciones familiares, roles de pareja, conflicto en la toma de decisiones, consenso diádico.

Abstract: Although the satisfaction with the tourist destination is an issue that has attracted
the interest of many researchers, it has not been so common in the case of family vacations. In
addition, there have been few works that collect the responses of men and women separately,
which allows a more exhaustive analysis of them. In this work, we have analyzed a sample of
375 couples, whose members answered separate questionnaires, being a considerable sample
size in family studies. It is concluded that, in general, when families travel, satisfaction with
the destination is usually high, without significant differences between sexes, although being
slightly higher in the case of women. The appearance of conflict between the couple during
the process of choosing the destination and the fact of traveling to a national or international
destination are the most influential variables in satisfaction, both for men and for women. The
practical implications indicate that the managers of the tourist destinations should be very
aware of the national tourists in their strategies, and should not ignore the use of rational

\footnotetext{
${ }^{1}$ Profesora Asociada, con el grado de doctora, del Departamento de Economía y Administración de Empresas, Universidad de Málaga, Andalucía Tech.mmrojasgracia@uma.es

${ }^{2}$ Catedrática de Escuela Universitaria, con el grado de doctora, del Departamento de Economía y

Administración de Empresas, Universidad de Málaga, Andalucía Tech.

pilar.alarcon@uma.es
} 
arguments in their communication campaigns to argue the convenience of the destination in case of appearance of conflict between the couple.

Key words: Satisfaction with the tourist destination, family vacations, couple roles, conflict in the decision making, dyadic consensus.

\section{INTRODUCCIÓN}

Durante los últimos años la literatura ha prestado una gran atención a la satisfacción del cliente, ya que se considera un antecedente de la fidelidad del cliente, e incluso del éxito empresarial (Reichheld y Sasser, 1990; Ostrowski et al. 1994; Teye y Leclerc, 1998). Sin embargo, dentro del ámbito turístico, estos estudios han sido más tardíos (Moreno Gil et al., 2002). No obstante, y a pesar de la importancia de tal concepto, aún no se ha llegado a conclusiones plenamente consensuadas entre los diferentes autores (Rojas-de Gracia y Alarcón-Urbistondo, 2016; 2017).

Por otro lado, aunque el concepto de vacaciones familiares no siempre está relacionado con la provisión de servicios, muchos investigadores consideran que implica un set de decisiones, que comienza normalmente con la elección del destino, con el principal objetivo de pasar un tiempo juntos, en un lugar diferente a aquel en el que la familia reside normalmente (van Raaij, 1986; Nichols y Snepenger, 1988; Kang y Hsu, 2004, 2005). Y, a pesar de la creciente influencia ejercida por los hijos en estas decisiones, existe un amplio consenso en que los principales decisores son los miembros de la pareja (Ritchie y Filiatrault, 1980; Belch et al., 1985; Ekstrom et al., 1986; Wang et al., 2004; Decrop y Snelders, 2005; Kozak y Karadag, 2012).

Es en este contexto en el que se va a desarrollar el trabajo que aquí se presenta, con el objetivo de analizar la satisfacción de la pareja con el destino de las vacaciones familiares. No es este un asunto baladí, dado que la satisfacción constituye un elemento clave para la consecución de los objetivos empresariales, al generarse los beneficios mediante la satisfacción de las necesidades de los consumidores (Kotler, 2000).

\section{MARCO TEÓRICO}

Aunque no existe una única definición de lo que se entiende por satisfacción, una de las más aceptadas es la de Kotler (2000). Según este autor, la satisfacción del cliente es el nivel del estado de ánimo de una persona que resulta al comparar el rendimiento percibido de un producto o servicio con sus expectativas. Se trata por tanto de un estado cognitivo derivado de la adecuación o inadecuación de la recompensa recibida respecto a la inversión realizada (Howard y Sheth, 1969).

Pese a que las vacaciones familiares están formadas por diferentes sub-decisiones (Rojas-de Gracia y Alarcón-Urbistondo, 2016), en la revisión de la literatura se encuentra que las familias, frecuentemente, han vinculado la satisfacción de las vacaciones en general, a la satisfacción con el destino, razón por la que ha resultado de gran interés para los investigadores turísticos (Cullinford, 1995; Nickerson y Jurowski, 2001; Kang y Hsu, 2005; Kozac y Kardag, 2012). En este sentido, los factores referidos al destino y a su proceso de decisión resultan fundamentales a la hora de identificar qué variables son las que intervienen en mayor medida en la satisfacción experimentada por las parejas. Entre estas variables se encuentran: (1) El tipo de destino; (2) La estructura de la decisión; (3) El grado de conflicto 
con la decisión del destino; y (4) El grado de consenso en la percepción de la pareja sobre la decisión del destino.

Respecto a la primera de ellas, es decir, al tipo de destino, son muchas las publicaciones que se han centrado en el estudio del turista internacional por considerar que tiende a mostrar comportamientos que los diferencia de los nacionales (Decrop, 2005; Carr, 2006; Nada et al., 2007; Hyde, 2008). Otros se han ocupado de diferenciar su comportamiento en función de la distancia al destino (Nicolau y Más, 2005; Hyde y Laesser, 2009). Es razonable pensar, por tanto, que el hecho de tratarse de un destino nacional o internacional puede influir en el grado de satisfacción.

En lo que se refiere a la estructura de la decisión, hay que destacar que la literatura del comportamiento de compra de las familias reconoce diferentes tipos de decisión en función de quién ejerza la mayor influencia (Davis y Rigaux, 1974; Litvin et al., 2004). Así, la decisión se denomina esposa-dominante si la influencia mayoritaria la ha ejercido la mujer; la decisión será marido-dominante si, por el contrario, la ha tenido el hombre; y será conjunta si la han ejercido ambos aproximadamente en la misma proporción. Kirchler (1995) postula que es menos probable que los miembros de la pareja tomen sus decisiones descuidando las preferencias del otro, debido a que el objetivo último es el de maximizar la utilidad conjunta. De forma similar, la teoría de la comparación social considera que los miembros de la familia evalúan sus propias preferencias a la luz de los sentimientos de los otros miembros (Kozak, 2010). Es por eso que es razonable pensar que la estructura de la decisión cuando se escogió el destino pueda influir en la satisfacción experimentada por la pareja con el mismo.

El tercer factor considerado, es decir, el grado de conflicto surgido durante el proceso de decisión es un factor que la literatura tradicionalmente ha relacionado con la satisfacción asociado a la compra de los bienes y servicios en particular y a las vacaciones en general (Bronner y de Hoog, 2008; Kang y Hsu, 2004).

Por último, el grado de consenso en la percepción que la pareja tiene sobre quién ha elegido el destino se refiere al hecho de que no siempre los miembros de la pareja coinciden en señalar a aquel de ellos que ha sido el que ha ejercido la mayor influencia y quien, por tanto, ha terminado escogiendo el destino (Rojas-de Gracia y Alarcón-Urbistondo, 2016). Así, mientras que uno de ellos puede pensar que realmente lo ha escogido él, el otro puede no haberlo percibido así. Considerando solo aquellas publicaciones de relevancia, se ha encontrado una única investigación que relaciona el grado de consenso en la percepción de la influencia ejercida y la satisfacción experimentada. Se trata del estudio de Bronner y de Hoog, (2008), quienes establecen que si el desacuerdo entre la pareja aumenta, disminuye la satisfacción experimentada por sus miembros.

En resumen, el esquema de variables predictivas de la satisfacción con el destino de las vacaciones es el que se representa en la figura 1.

FIGURA 1.

influyen en la destino

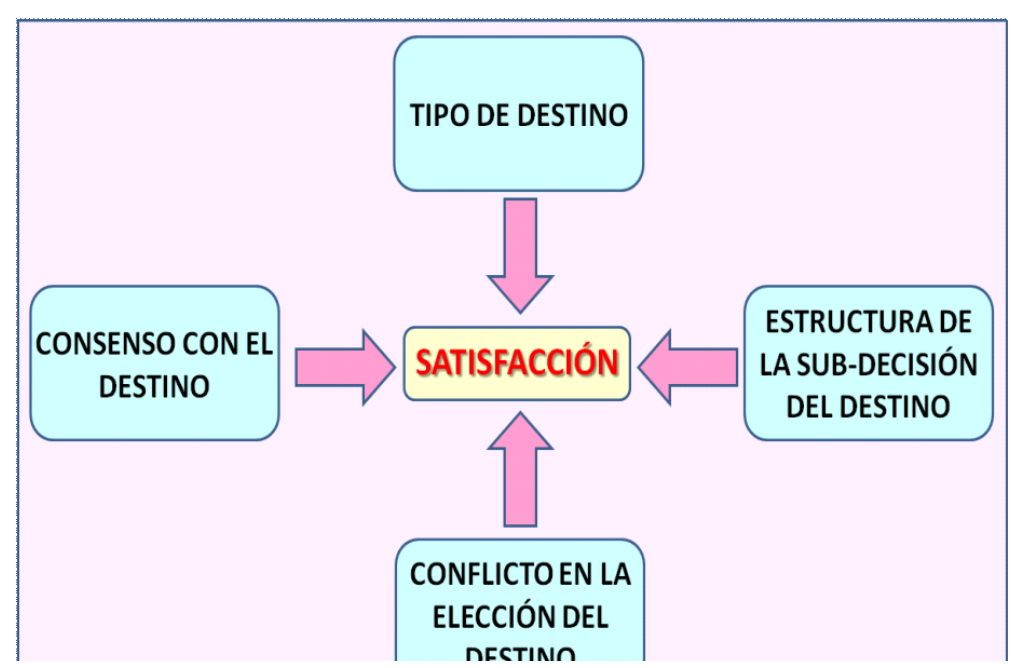

Variables que satisfacción con el 


\section{METODOLOGÍA}

Fuente: Elaboración propia.

\subsection{Muestra}

La población objeto de estudio para este trabajo, que forma parte de una investigación más amplia, está formada por parejas heterosexuales que realizaron un viaje vacacional en el que al menos un hijo común, de entre 10 y 18 años, les acompañara. Es decir, se ha considerado pareja a la diada compuesta por un hombre y una mujer que conviven juntos. Por otro lado, se ha considerado viaje vacacional si pasaron al menos tres días fuera de casa con el propósito principal de ocio (Etzel y Whlers, 1985; Gitelson y Crompton, 1984). El hecho de incluir a los hijos en el grupo de viaje responde a que el objeto de estudio son los viajes familiares en los que las decisiones de la pareja no les afectan únicamente ellos, sino también a sus hijos.

Para poder determinar si los miembros de la pareja coincidían en sus respuestas, se les preguntó a hombres y mujeres por separando, obteniendo finalmente una muestra de 375 diadas de cuestionarios. El hecho de contar con las dos respuestas constituye una aportación interesante, al ser una técnica poco utilizada hasta la fecha, dada la mayor dificultad de tener que recoger dos respuestas por hogar.

\subsection{Recogida de datos}

Se repartieron cuestionarios a los alumnos de varios centros educativos públicos y privados de España, de edades comprendidas entre los 10 y los 18 años, que debían hacer llegar a sus padres. Estos cuestionarios iban precedidos por una carta en la que se les hacía hincapié en la importancia de que ambos progenitores los rellenasen por separado.

Se repartieron un total de 1.200 diadas de cuestionarios, de las que se recogieron 536, siendo $375(31,3 \%)$ las que finalmente fueron útiles. La ausencia de respuestas válidas ha tenido varias causas, siendo la más frecuente las incongruencias entre las respuestas de cada miembro de la pareja cuando se les preguntaba por aspectos en los que debían coincidir, como el destino, y la entrega de encuestas incompletas. Por otra parte, el problema de la no respuesta, es un problema frecuente y difícilmente subsanable, sobre todo teniendo en cuenta que se solicitaban dos respuestas por hogar. La única manera de paliarlo es la entrevista con el fin de cotejar si la esta falta de respuesta apunta mayoritariamente en un sentido. Esta ha sido la solución llevada a cabo en este estudio. Así, las observaciones hechas por los tutores de los diferentes cursos escolares en los que se repartió el cuestionario constatan que la falta de colaboración se ha debido a una cuestión de desidia por parte de padres y/o hijos, que no tiene porqué provocar ningún tipo de sesgo sistemático, que sería lo más problemático.

En cuanto al cuestionario, este fue validado, en primer lugar, por un grupo de 6 expertos del ámbito tanto académico como profesional. Posteriormente se llevó a cabo un pretest que respondieron 30 parejas. Finalmente, el cuestionario resultante, aparte de recopilar 
datos socio-demográficos, de comportamiento de viaje (tabla 1), preguntó directamente por la percepción de la influencia ejercida por cada uno de los miembros de la pareja a la hora de decidir el destino (tabla 2), tal y como es frecuente en este tipo de estudios (Madrigal y Miller, 1996). En concreto, se les ofreció a los encuestados la posibilidad de responder "principalmente yo", "principalmente mi pareja", "los dos" u "otros/ninguno". Se incluyó esta última opción para no forzarles a tener que elegir entre ellos. En tercer lugar, se les pidió que indicasen el grado de conflicto durante el proceso de decisión (tabla 3). Por último, se les pidió que indicasen el grado de satisfacción con el destino escogido (tabla 4).

TABLA 1. Datos socio-demográficos y de comportamiento de viaje de la muestra

\begin{tabular}{lcc}
\hline Variables & Frecuencia & Válidos \% \\
\hline Situación laboral de la mujer & & \\
Mujer no trabajadora & 140 & 51.85 \\
Mujer trabajadora & 130 & 48.15 \\
Diferencia de estudios & \multicolumn{2}{c}{} \\
$\quad$ Él más que ella & 62 & 16.67 \\
Ella más que él & 98 & 26.34 \\
Mismo nivel educativo & 212 & 56.99 \\
Tiempo de convivencia & & \\
Menos de 20 años & 154 & 41.07 \\
20 años o más & 221 & 58.93 \\
Tipo de destino & & \\
Nacional & 285 & 76.20 \\
Internacional & 89 & 23.80 \\
Motivo de las vacaciones & & \\
Exclusivamente ocio & 306 & 81.82 \\
Visita familiares y amigos & 57 & 15.24 \\
Otros & 11 & 2.94 \\
Frecuencia de las vacaciones & & \\
Esporádicamente & 201 & 53.60 \\
Cada dos o tres años & 94 & 25.07 \\
Una vez al año & & 21.33 \\
\hline
\end{tabular}

TABLA 2. Influencia de los miembros de la pareja en la elección del destino

\begin{tabular}{lrrrrrr}
\hline & \multicolumn{3}{c}{ Según hombre } & \multicolumn{3}{c}{ Según mujer } \\
& Frec. & \% & \% Válido & Frec. & \% & \% Válido \\
\hline Marido-dominante & 47 & 12,53 & 13,54 & 42 & 11,20 & 12,10 \\
Esposa-dominante & 68 & 18,13 & 19,60 & 69 & 18,40 & 19,88 \\
Conjunta & 232 & 61,87 & 66,86 & 236 & 62,93 & 68,01 \\
No lo decidieron & 25 & 6,67 & - & 26 & 7,49 & - \\
ellos & 3 & 0,80 & - & 2 & 0,58 & - \\
Perdidos & 375 & 100 & 100 & 375 & & 100
\end{tabular}


TABLA 3. Grado de conflicto de los miembros de la pareja en la elección del destino

\begin{tabular}{lrrrrrr}
\hline & \multicolumn{3}{c}{ Según hombre } & \multicolumn{3}{c}{ Según mujer } \\
& Frec. & $\%$ & \% Válido & Frec. & \% & \% Válido \\
\hline Sí & 192 & 51,20 & 51,61 & 197 & 52,53 & 56,77 \\
No & 155 & 41,33 & 48,39 & 150 & 40,00 & 43,23 \\
No lo decidieron & 25 & 6,67 & - & 26 & 7,49 & - \\
ellos & 3 & 0,80 & - & 2 & 0,58 & - \\
$\quad$ Perdidos & 375 & 100 & 100 & 375 & & 100
\end{tabular}

TABLA 4. Grado de satisfacción con el destino

\begin{tabular}{lrrrrrr}
\hline & \multicolumn{3}{c}{ Según hombre } & \multicolumn{4}{c}{ Según mujer } \\
& Frec. & $\%$ & \% Válido & Frec. & $\%$ & \% Válido \\
\hline Nada & 2 & 0,53 & 0,58 & 2 & 0,53 & 0,58 \\
Poco & 9 & 2,4 & 2,59 & 7 & 1,87 & 2,02 \\
Bastante & 154 & 41,07 & 44,38 & 154 & 41,06 & 44,08 \\
Mucho & 181 & 48,26 & 52,16 & 179 & 47,73 & 51,59 \\
No lo decidieron & 25 & 6,67 & - & 26 & 7,49 & - \\
ellos & 3 & 0,80 & - & 2 & 0,58 & - \\
Perdidos & 375 & 100 & 100 & 375 & & 100
\end{tabular}

\subsection{Análisis de los datos}

Para la consecución del objetivo propuesto en este trabajo, es decir, la identificación de aquellas características del destino y de su proceso de decisión que influyen en la satisfacción con el mismo se ha utilizado la segmentación jerárquica, también conocida como técnica de árboles. Esta es una técnica que se incluye dentro de los métodos multivariantes de dependencia, puesto que hace distinción entre variables explicativas y variables a explicar. Se trata de un conjunto de algoritmos utilizados tradicionalmente en el ámbito de la investigación de mercados para el análisis, entre otras muchas variables, de la satisfacción (Luque, 2000). En concreto, se utiliza el algoritmo CHAID (Chi-Squared Automatic Interaction Detection) que requiere una variable dependiente categórica (en este caso la satisfacción con el destino) y varias variables independientes o predictivas que, combinadas, permiten identificar segmentos. El algoritmo deja de hacer divisiones entre los grupos si no encuentra diferencias significativas entre las variables que caracterizan a los integrantes.

La razón de utilizar esta técnica es que el objetivo que se busca no se limita simplemente a describir grupos, como haría un análisis cluster, ya que no propicia que se obtengan los grupos en función de determinados criterios. Con la segmentación jerárquica se pretende obtener segmentos que vengan definidos explícitamente por combinaciones de variables independientes predictivas.

\section{RESULTADOS}

\subsection{Comparación de la satisfacción de la pareja con las vacaciones familiares}

En primerlugar, según los resultados ofrecidos en la tabla 4, se observa que en el nivel de satisfacción de los miembros de la pareja es muy elevado, siendo muy pocos los casos en 
los que los encuestados se encontraron poco o nada satisfechos (3,2\% según la muestra de los hombres -en adelante s.m.h.- y 2,6\% según la muestra de las mujeres -en adelante s.m.m-).

Las diferencias de satisfacción se pueden estudiar a nivel agregado o a nivel de pareja. A nivel agregado se comparan las medias obtenidas por cada uno de los sexos, teniendo en cuenta que su puntuación puede ir de 1 a 4, es decir, desde nada satisfecho a muy satisfecho. En este caso, la media de las mujeres fue ligeramente mayor que la de los hombres, aunque sin encontrar diferencias significativas entre sexos (3,47; D.E.: 0,593 s.m.h. y 3,49; D.E.:0,571 s.m.m [p=0,963]). A nivel de pareja, el porcentaje de casos en los que ambos miembros tuvieron el mismo nivel de satisfacción fue de un 69,6\% (tabla 5). Dentro de los casos en los que existieron diferencias, fue ligeramente mayor el número de parejas en las que ella se sintió más satisfecha que él, que en el caso contrario, aunque sin presentar diferencias significativas $(16,0 \%$ Vs. $13,3 \%, p=0,298)$.

\begin{tabular}{lrrr}
\multicolumn{4}{c}{ TABLA 5. Diferencias en el grado de satisfacción con el destino } \\
\hline & Frec. & $\%$ & \% Válido \\
\hline Mismo nivel de satisfacción & 261 & 69,60 & 70,70 \\
Él más satisfecho que ella & 49 & 13,10 & 13,30 \\
Ella más satisfecha que él & 59 & 15,70 & 16,00 \\
Perdidos & 6 & 1,60 & - \\
\hline TOTAL & 375 & 100 & 100
\end{tabular}

A nivel de parejas (tabla 6), el mayor consenso entre las respuestas de los integrantes de la pareja se dio cuando ambos afirmaron sentirse muy satisfechos $(39,0 \%)$ de los casos, seguido de aquellos en los que ambos se consideraron bastante satisfechos $(30,6 \%)$. Le siguen en importancia las parejas en las que la mujer declaró estar muy satisfecha y el hombre bastante $(13,8 \%)$, y el caso contrario supuso un porcentaje ligeramente menor (11,9\%). Las demás combinaciones constituyeron el $4,7 \%$ de los casos.

TABLA 6. Diferencias en el grado de satisfacción con el destino

\begin{tabular}{|c|c|c|c|c|c|c|}
\hline & & \multicolumn{4}{|c|}{ Satisfacción de la mujer } & \multirow[t]{2}{*}{ Total } \\
\hline & & Nada & Poco & Bastante & Mucho & \\
\hline \multirow{4}{*}{ Satisfacción del hombre } & Nada & $1(0,3)$ & $1(0,3)$ & $1(0,3)$ & $0(0,0)$ & $3(0,8)$ \\
\hline & Poco & $1(0,3)$ & $3(0,8)$ & $6(1,6)$ & $0(0,0)$ & $10(2,7)$ \\
\hline & Bastante & $0(0,0)$ & $3(0,8)$ & $113(30,6)$ & $51(3,8)$ & $167(45,3)$ \\
\hline & Mucho & $0(0,0)$ & $1(0,3)$ & $44(11,9)$ & $144(39,0)$ & $189(51,2)$ \\
\hline Total & & $2(0,5)$ & $8(2,2)$ & $64(44,4)$ & $195(52,8)$ & $\begin{array}{c}369 \\
(100,0)\end{array}$ \\
\hline
\end{tabular}

Nota: Dentro de las casillas aparece el recuento total y el \% del total entre paréntesis

\subsection{Segmentación jerárquica de la pareja según su satisfacción con el destino}

El objetivo de esta parte del trabajo es realizar una segmentación jerárquica para llegar a la delimitación e identificación de subconjuntos homogéneos de los miembros de la pareja, distinguiendo por sexos, con arreglo a su satisfacción con el destino de las vacaciones, considerando ciertas características ligadas al mismo. Debido al tamaño limitado de las dos muestras de las que se dispone, se utilizará esta técnica con carácter meramente exploratorio, 
estableciendo en 30 el mínimo de casos en un nodo parental, tal y como realiza Chen (2003) en una publicación con características similares a las del presente trabajo.

Dado que el porcentaje de respuestas entre los que se consideraron bastante y muy satisfechos supuso el $96,8 \%$ de la muestra en el caso de los hombres y $97,3 \%$ en el de las mujeres (tabla 4), se ha optado por distinguir entre estos dos grupos sin considerar aquellos que estaban poco o nada satisfechos, debido a su escasa frecuencia. Por este motivo se ha realizado la segmentación entre los que contestaron estar bastante satisfechos o muy satisfechos, siendo esta última la categoría objetivo.

\subsection{Segmentación jerárquica de los hombres según su satisfacción con el destino}

En la figura 2 se presenta el árbol de segmentación para la muestra de los hombres. Los resultados del algoritmo CHAID muestran que los hombres que viajan en familia y que declararon sentirse bastante o muy satisfechos con el destino se pueden dividir en cuatro segmentos. Del nodo raíz o nodo 0 surge la muestra a dividir, compuesta de 335 hombres, de los que el $46 \%$ manifestaron sentirse bastante satisfechos y el $54 \%$ muy satisfechos o satisfechos al máximo nivel.

El mejor predictor, por tanto, de la satisfacción con el destino ha resultado ser el conflicto en su elección, variable para la que se han encontrado diferencias significativas $(\mathrm{p}<0,001)$ entre los que manifestaron algún grado de conflicto y los que no. El grupo que experimentó algún conflicto está formado por 148 individuos, de los que un 41,9\% declararon estar muy satisfechos. Este nodo es terminal, al no derivarse de él ninguno más, ya que o bien no es significativo o bien no cumple el requisito relativo al criterio que se le impuso de no formar grupos menores de 30 casos. Constituye el segmento 1, que se corresponde con el nodo 1 .

En el grupo que no tuvo ningún conflicto en la elección del destino, formado por 187 individuos, el porcentaje que se sintió muy satisfecho fue mayor que en el que sí lo hubo, en concreto un 63,6\%. De este nodo, considerado padre porque de ellos derivan otros, resultan otros nodos hijos. Para aquellos que manifestaron no haber tenido ninguna dificultad con la decisión del destino, la variable en la que se encuentran diferencias significativas es el tipo de destino $(p=0,010)$. Entre aquellos individuos que no encontraron conflicto con el destino y eligieron un destino nacional, se encontraron muy satisfechos en el 59,3\% de los casos, mientras que, en este mismo caso, los que viajaron a un destino internacional se sintieron muy satisfechos un $81,1 \%$, conformando este último grupo el segmento 4 (nodo 4), ya que es un nodo terminal. Este último segmento, a pesar de ser el grupo con el menor número de individuos, es el que presenta un mayor porcentaje de individuos muy satisfechos.

En el caso de los hombres que no experimentaron ningún conflicto y viajaron a un destino nacional, la mejor variable predictiva fue la estructura de la decisión al escoger el destino. Cuando fue autónoma, un $41,7 \%$ de ellos se encontraron muy satisfechos. Este grupo conforma el segmento 2 (nodo 5). Pero cuando fue conjunta, este porcentaje aumenta a un $64,9 \%$, compuesto por 74 individuos. Es lo que se ha identificado como el segmento 3 (nodo $6)$.

En resumen, los segmentos encontrados en la muestra de los hombres se definen así:

Segmento 1: Turistas que experimentaron algún conflicto con sus parejas en la elección del destino. 
Segmento 2: Turistas que no experimentaron ningún conflicto con sus parejas en la elección del destino, viajaron a un destino nacional y tomaron la decisión de forma autónoma.

Segmento 3: Turistas que no experimentaron ningún conflicto con sus parejas en la elección del destino, viajaron a un destino nacional y tomaron la decisión de forma conjunta entre ellos.

Segmento 4: Turistas que no experimentaron ningún conflicto con sus parejas en la elección del destino y viajaron a un destino nacional.

La tabla de ganancia de la categoría objetivo, es decir, los que están muy satisfechos, se presenta en la tabla 7.

FIGURA 2. Segmentación jerárquica de la satisfacción de los hombres 
Satiśacción

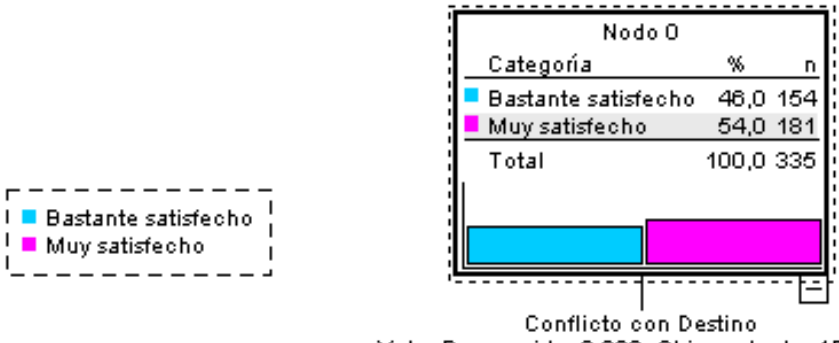

Valor P corregido $=0,000$, Chi-cuadrado $=15,818$,

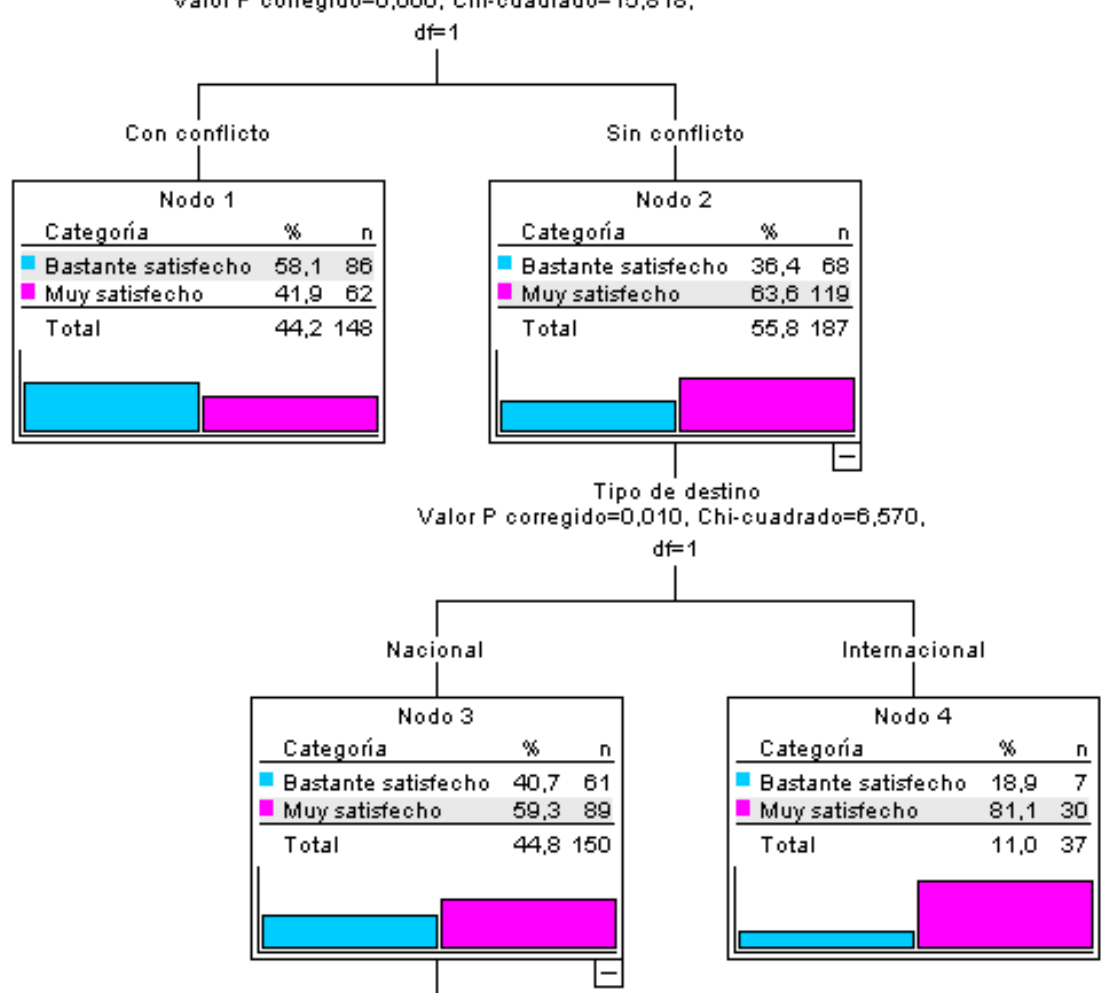

Estructura decisión destino

Valor P corregido $=0,042$, Chi-cuadrado $=6,043$.

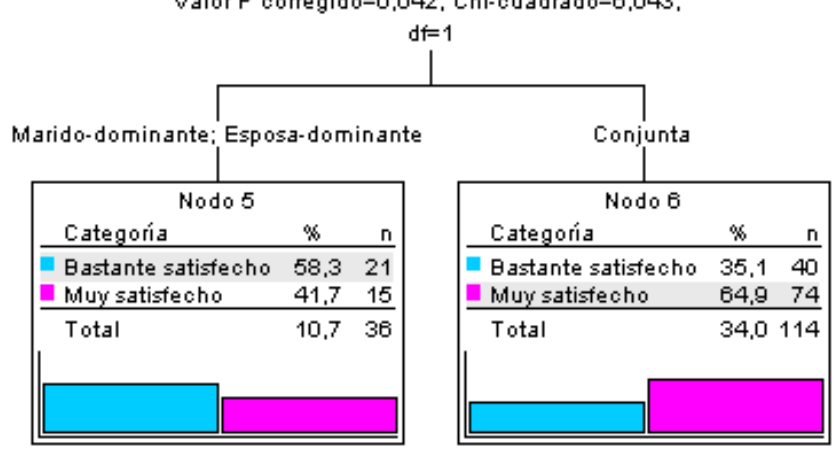


TABLA 7. Tabla de ganancia para los segmentos en la muestra de hombres

\begin{tabular}{cccccccc}
\hline \multirow{2}{*}{ Segmento } & Nodo & \multicolumn{2}{c}{$\begin{array}{c}\text { Nodo } \\
\text { Porcentaje }\end{array}$} & $\mathrm{N}$ & $\begin{array}{c}\text { Ganancia } \\
\text { Porcentaje }\end{array}$ & Respuesta & Índice \\
\hline 4 & 4 & 37 & $11,0 \%$ & 30 & $16,6 \%$ & $81,1 \%$ & $150,1 \%$ \\
3 & 6 & 114 & $34,0 \%$ & 74 & $40,9 \%$ & $64,9 \%$ & $120,1 \%$ \\
1 & 1 & 148 & $44,2 \%$ & 62 & $34,3 \%$ & $41,9 \%$ & $77,5 \%$ \\
2 & 5 & 36 & $10,7 \%$ & 15 & $8,3 \%$ & $41,7 \%$ & $77,1 \%$ \\
\hline
\end{tabular}

Métodos de crecimiento: CHAID

Variable dependiente: Satisfacción

El segmento con mayor porcentaje de ganancia (40,9\%) es el 3. Esto significa que el grupo que incluye a un mayor número de turistas muy satisfechos es el que está formado por aquellos que no experimentaron ningún conflicto con sus parejas en la elección del destino, viajaron a un destino nacional y tomaron la decisión de forma conjunta entre ellos.

Las variables predictivas de la satisfacción con el destino de los hombres han sido, por tanto, el conflicto con la elección del destino, el tipo de destino al que se viaja y la estructura de su decisión. Por el contrario, el consenso en la percepción de la influencia ejercida en la elección del destino no ha resultado ser una variable significativa. Para confirmarlo se ha realizado un análisis mediante tablas de contingencia con la satisfacción y el consenso y no se ha podido rechazar la hipótesis nula de independencia entre ellas $(\mathrm{p}=0,298)$.

Los segmentos 4 y 3 son los denominados segmentos accionables. Son los que presentan un mayor interés para los responsables de la gestión del marketing de las empresas turísticas, dado el alto grado de satisfacción de sus componentes y sus mayores posibilidades de recomendación del destino a otros (Kozak, 2010). Presentan un índice de ganancia superior a $100 \%$, es decir, superior al general de la muestra, ya que este índice compara la ganancia de cada grupo respecto a la media.

Con el objetivo de comprobar si existen características socio-demográficas que puedan identificar a los hombres pertenecientes a un segmento accionable, se realizan análisis mediante tablas de contingencia. Se encontró una relación significativa con la variable "situación laboral de la mujer" ( $\mathrm{p}=0,018)$. Según este análisis, cuando en la pareja la mujer no trabaja, la probabilidad de que el hombre pertenezca a un segmento accionable es 1,69 veces mayor (IC95\%: 1,09-2,63) que cuando ella sí trabaja en la pareja.

\subsection{Segmentación jerárquica de las mujeres según su satisfacción con el destino}

El árbol de segmentación para la muestra de mujeres se presenta en la figura 3. Los resultados del algoritmo CHAID indican que las mujeres que viajan en familia y declararon sentirse bastante o muy satisfechas se pueden dividir en tres segmentos. Del nodo raíz surge la muestra a dividir, compuesta por 333 mujeres, de las que el 46,2\% manifestaron sentirse bastante satisfechas y el 53,8\% muy satisfechas. No se hallaron diferencias significativas de estos porcentajes respecto a la muestra de los hombres $(\mathrm{p}=0,877)$.

El mejor predictor de la satisfacción con el destino en el caso de las mujeres es el tipo de destino, mostrando diferencias significativas $(\mathrm{p}<0,001)$ entre las mujeres que viajaron a un destino nacional o internacional. Se observa en el árbol de segementación que al grupo que viajó a un destino internacional se les añaden los datos perdidos, aunque se comprueba que se 
trata de un único caso de los 75 que lo conforman. Las turistas que viajaron al extranjero forman el segmento 1 (nodo 2), representado por un nodo terminal y, por tanto, sin más divisiones. En este segmento, el 73,3\% de las mujeres declararon estar satisfechas al máximo nivel. A pesar de ser el segmento con menor número de individuos, es el que presenta un mayor porcentaje de mujeres satisfechas en su grado máximo.

En el grupo que viajó a un destino nacional, formado por 258 mujeres, el porcentaje que se sintieron muy satisfechas fue menor que el grupo con destino internacional, representando un $(73,3$ vs $48,1 \%)$. A su vez, este grupo se divide en otros, en función del conflicto surgido en la elección del destino. De esta forma, aquellas mujeres que experimentaron alguna dificultad y eligieron un destino nacional se encontraron muy satisfechas en un $36,1 \%$ de los casos. En cambio, aquellas que, habiendo viajado también a un destino nacional, no apreciaron ningún conflicto al escoger el destino se sintieron muy satisfechas en el 56,7\% de los casos. El primero de los grupos forma el segmento 2 (nodo 3), mientras que el segundo forma el segmento 3 (nodo 4). siguientes:

En síntesis, los segmentos identificados en la muestra de las mujeres son los Segmento 1: Turistas que viajaron a un destino internacional.

Segmento 2: Turistas que viajaron a un destino nacional y experimentaron algún conflicto con sus parejas en la elección del destino.

Segmento 3: Turistas que viajaron a un destino nacional y no experimentaron ningún conflicto con sus parejas en la elección del destino.

La tabla de ganancia de la categoría objetivo, que son las mujeres que estuvieron muy satisfechas, se presenta en la tabla 9. El segmento que presenta mayor porcentaje de ganancia $(47,5 \%)$ es el número 3. Esto significa que el grupo más numeroso de mujeres muy satisfechas fueron las que viajaron a un destino nacional y no experimentaron ningún conflicto con sus parejas en la elección del destino.

Ni la estructura de la decisión del destino ni el consenso entre la pareja respecto a la percepción de la influencia en la elección del destino han resultado ser variables significativas. Para confirmarlo se han realizado sendos análisis mediante tablas de contingencia, comparando la satisfacción con cada una de estas dos variables. En el caso de la variable relacionada con la estructura en la elección del destino, aunque presentó una relación significativa con la satisfacción, no aparece como predictiva, por ser su significación menor que las variables del tipo de destino y el conflicto en su elección, que sí son susceptibles de predecir la satisfacción de las mujeres con el destino según el árbol de decisión $(p<0,001$ vs $p=0,003$ y $p=0,001$ vs $p=0,003$, respectivamente). Sin embargo, para la variable relacionada con el consenso no se puede rechazar la hipótesis nula de independencia con el nivel de satisfacción $(\mathrm{p}=0,357)$.

Los segmentos 1 y 3 son los segmentos accionables, mientras que el segmento 2 no lo es. Al igual que se hizo para la muestra de los hombres, se realizaron sendos análisis mediante tablas de contingencia entre cada una de las variables socio-demográficas y el hecho de pertenecer a un segmento accionable sin que en este caso se encontraran asociaciones significativas. 
FIGURA 3. Segmentación jerárquica de la satisfacción de las mujeres

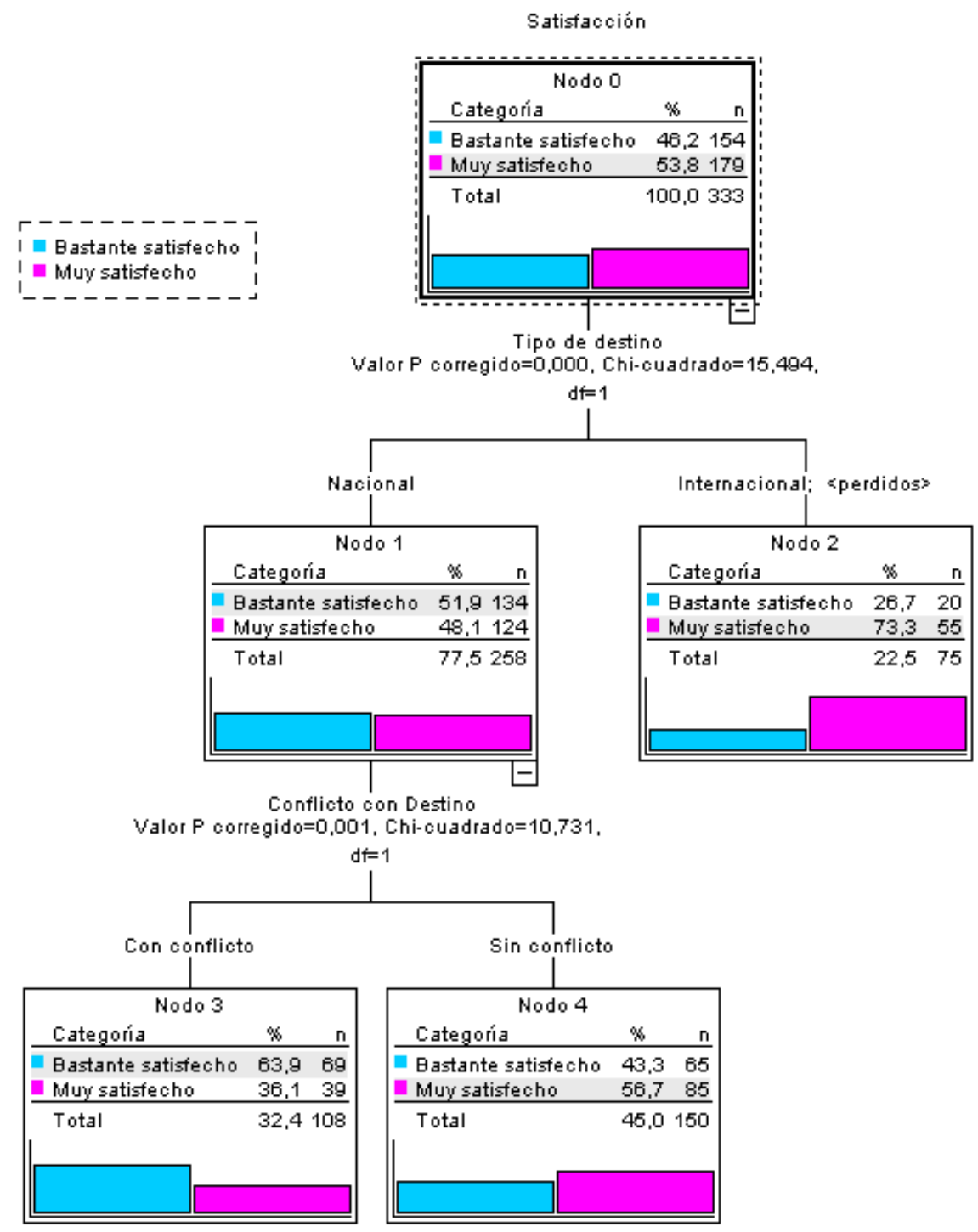

TABLA 9. Tabla de ganancia para los segmentos en la muestra de hombres

\begin{tabular}{|c|c|c|c|c|c|c|c|}
\hline \multirow{2}{*}{ Segmento } & \multirow{2}{*}{ Nodo } & \multicolumn{2}{|c|}{ Nodo } & \multicolumn{2}{|c|}{ Ganancia } & \multirow{2}{*}{ Respuesta } & \multirow{2}{*}{ Índice } \\
\hline & & $\mathrm{N}$ & Porcentaje & $\mathrm{N}$ & Porcentaje & & \\
\hline 1 & 2 & 75 & $22,5 \%$ & 55 & $30,7 \%$ & $73,3 \%$ & $136,4 \%$ \\
\hline 3 & 4 & 150 & $45,0 \%$ & 85 & $47,5 \%$ & $56,7 \%$ & $105,4 \%$ \\
\hline 2 & 3 & 108 & $32,4 \%$ & 39 & $21,8 \%$ & $36,1 \%$ & $67,2 \%$ \\
\hline
\end{tabular}

Métodos de crecimiento: CHAID

Variable dependiente: Satisfacción 


\section{CONCLUSIONES, DISCUSIÓN E IMPLICACIONES}

Este trabajo ha puesto de manifiesto que el grado de satisfacción que experimentaron las parejas con el destino seleccionado fue muy elevado, sin mostrar grandes diferencias entre sus miembros. De hecho, en más del $96 \%$ de los casos, los miembros de la pareja se sintieron o bastante o muy satisfechos. No obstante, al igual que los resultados obtenidos por Kang y Hsu (2005), las mujeres están ligeramente más satisfechas que los hombres, consideradas conjuntamente como grupo y también al comparar su satisfacción con la de su compañero.

Cuando se analizan los factores relevantes que intervienen en la satisfacción experimentada según las características del destino y de su proceso de decisión, se ha puesto de manifiesto que no experimentar ningún conflicto en la elección del destino y viajar al extranjero son factores que afectan positivamente a la satisfacción con el destino. Sin embargo, estas dos circunstancias no afectan con la misma intensidad a la satisfacción de los hombres y las mujeres. En tanto que para ellos la aparición de conflicto en la elección del destino resulta ser el principal factor determinante de su satisfacción con el mismo, para ellas es más relevante haber viajado a un destino internacional.

La relación entre la satisfacción y la presencia de conflicto observada en el presente trabajo contradice lo que afirmaron Kang y Hsu (2004), indicando que la presencia de conflicto en la elección del destino entre los miembros de la pareja no hace decrecer necesariamente el nivel de satisfacción. No obstante, parece lógico pensar que la existencia de conflicto a la hora de escoger el destino implica que al menos uno de los miembros de la pareja tuvo que ceder, en contra de sus preferencias individuales, afectando así a su satisfacción.

Por otro lado, la relación entre la satisfacción con el destino y el hecho de que este sea nacional o internacional ha sido una cuestión escasamente estudiada con anterioridad. En cambio, ha sido más frecuente comparar, para un destino en concreto, la satisfacción experimentada por los turistas que provienen de otros países con la de los nacionales, como en el caso de Tailandia, en el que los turistas internacionales estuvieron más satisfechos que el turista doméstico (Mechinda et al.; Tidtichumrernporn et al., 2010).

La explicación a esta mayor satisfacción con los destinos internacionales podría radicar en la teoría de la disonancia cognitiva, que afirma que cuando existen discrepancias entre las expectativas creadas para un producto y su rendimiento, se genera una tensión psicológica que lleva al consumidor a minimizar la desviación, haciendo su percepción del producto más consistente con las expectativas (Valera, 1992). Debido a que es lógico pensar que los destinos internacionales generan unas expectativas mayores al requerir generalmente un mayor desembolso económico (Hyde y Decrop, 2011), en los casos en los que el destino no ha cubierto las expectativas podría existir una tendencia a sobrestimar la satisfacción que tal destino ha reportado.

Asimismo, algunos autores han sugerido que los turistas con aversión al riesgo, especialmente los que quieren reducir su riesgo de insatisfacción, cuando escogen el destino tienen como criterio la familiaridad con el lugar, por lo que permanecen en algún destino familiar, incluso si no están del todo satisfechos (Giltenson y Crompton, 1984; Mechinda et al., 2009; Oppermann, 1998). Sin embargo, son necesarios más estudios al respecto que confirmen estas posibles explicaciones.

La forma de elección del destino, es decir, que se decida conjuntamente o no, repercute en la satisfacción experimentada principalmente en el caso de los hombres, que se 
encontraron más satisfechos si lo escogieron conjuntamente con su pareja, lo que coincide con las publicaciones de Kirchler (1995) y Kozak (2010).

En cambio, no se ha podido demostrar la existencia de una relación entre el consenso entre los miembros de la pareja sobre la percepción de la influencia ejercida cuando se escogió el destino y la satisfacción asociada al mismo, a pesar de lo postulado por Bronner y de Hoog (2008). Según estos autores, si la pareja no coincide en la percepción sobre quien ejerció mayor influencia, es decir, si existió desacuerdo diádico, disminuye la satisfacción con las vacaciones experimentada por los miembros de la pareja.

Estos hallazgos tienen implicaciones prácticas para los gestores de los destinos. En primer lugar, tienen que intentar satisfacer las necesidades no solamente de los turistas internacionales, sino también las de los nacionales, dado que en muchas ocasiones estos últimos experimentan un grado de satisfacción menor. De igual forma, en las campañas de comunicación de marketing se deberían dar argumentos que puedan utilizarse como posibles soluciones que, en grado de aparecer conflicto en la elección del destino, refuercen la sensación de acierto sobre la decisión tomada. En este sentido, se debería apelar no solo a los sentimientos, sino que también se deberían ofrecer argumentos racionales.

\section{BIBLIOGRAFÍA}

BELCH, G. E.; BELCH, M. A.; CERESINO, G. Parental and teenage child influences in family decision making. En:Journal of Business Research. 1985, V.13, no. 2, pp. 163176.

BRONNER, F.; DE HOOG, R.Agreement and disagreement in family vacation decisionmaking. En:Tourism Management.2008, V. 29, no. 5, pp. 967-979.

CARR, N. A comparison of adolescents' and parent's holiday motivations and desires. En Tourism and Hospitality Research. 2006, V. 6, no. 2, pp. 129-142.

CHEN, J. S. Market segmentation by tourists' sentiments. En:Annals of Tourism Research. 2003, V. 30, no. 1, pp. 178-193.

CULLINGFORD, C.Children's attitudes to holidays overseas. En:Tourism Management, 1995, V. 16, no. 2, pp. 121-127.

DAVIS, H. L.; RIGAUX, B. P.Perception of marital roles in decision processes. En:Journal of Consumer Research. 1974,V. 1, no. 1, pp. 51-62.

DECROP, A.; SNELDERS, D.A grounded typology of vacation decision-making. En:Tourism Management. 2005, V. 26, no. 2, pp. 121-132.

EKSTROM, K. M.; TANSUHAJ, P. S.; FOXMAN, E. R.Children's influence in family decisions and consumer socialization: A reciprocal view. En:Advances in Consumer Research.1986, V. 13, pp. 283-288.

ETZEL, M. J.; WAHLERS, R. G.The use of requested promotional material by pleasure travelers. En:Journal of Travel Research. 1985, V. 23, no. 4, pp. 2-6.

GITELSON, R. J.; CROMPTON, J. L. Insights into the repeat vacation phenomenon. En:Annals of Tourism Research. 1984, V. 11, no. 2, pp. 199-217.

HOWARD, D.; SHETH, J. N.The theory of buyer behavior, New York: John Wiley, 1969 
HYDE, K.Information processing and touring planning theory. En:Annals of Tourism Research. 2008, V. 35 no. 3, pp. 712-731.

HYDE, K.F.; DECROP, A.New perspectives on vacation decision making. En:International Journal of Culture Tourism and Hospitality Research. 2011, V. 5, no. 2, pp. 103-111.

HYDE, K. F.; LAESSER, C.A structural theory of the vacation. En:Tourism Management. 2009, V. 30, no. 2, pp. 240-248.

KANG, S. K.; HSU, C. H. C.Spousal conflict level and resolution in family vacation destination selection. En:Journal of Hospitality \& Toursim Research. 2004, V. 28, no. 4, pp. 408-424.

KANG, S. K.; HSU, C. H. C.Dyadic consensus on family vacation destination selection. En:Toursim Management. 2005, V. 26, no. 4, pp. 571-582.

KIRCHLER, E.Studying economic decisions within private households: A critical review and design for a "couple experiences diary". En: Journal of Economic Psychology. 1995, V. 16, pp. 393-419.

KOTLER, P.Marketing management, millenium edition, Tenth edition, New Jersey: Upper Saddle River,2000.

KOZAK, M.Holiday taking decisions - The role of spouses. En:Tourism Management.2010, V. 31, no. 4, pp. 489-494.

KOZAK, M.; KARADAG, L.Who influences aspects of family decision making?. En:International Journal of Culture, Tourism and Hospitality Research.2012, V. 6, no. 1, pp. 8-20.

LITVIN, S. W., XU, G., KANG, S. K.Spousal vacation-buying decision making revisited across time and place. En:Journal of Travel Research. 2004, V. 43, no. 2, pp. 193-198.

LUQUE MARTÍNEZ, T. Técnicas de análisis de datos en investigación de mercados, Madrid: Pirámide, 2000.

MADRIGAL, R.; MILLER, C. M.Construct validity of spouses' relative influence measures: An application of the direct product model. En:Journal of the Academy of Marketing Science.1996, V. 24, no. 2, pp. 157-170.

MECHINDA, P.; SERIRAT, S; GUILD, N.An examination of tourists' attitudinal and behavioral loyalty: Comparison between domestic and international tourists. En:Journal of Vacation Marketing. 2009, V. 15, pp. 129-148.

MORENO GIL, S.; CELIS SOSA, D. F.; AGUILAR QUINTANA, T.Análisis de la satisfacción del turista de paquetes turísticos respecto a las actividades de ocio en el destino: El caso de República Dominicana. En:Cuadernos de Turismo. 2002, V. 9, pp. 67-84.

NANDA, D., HU, C.; BAI, B.:Exploring family roles in purchasing decisions during vacation planning. En:Journal of Travel \& Tourism Marketing. 2007, V. 20, no. 3-4, pp. 107125.

NICHOLS, C. M.; SNEPENGER, D. J.Family decision making and tourism behavior and attitudes. En:Journal of Travel Research. 1988, V. 26, no. 4, pp. 2-6. 
NICKERSON, N. P.; JUROWSKI, C. The influence of children on vacation travel patterns. En: Journal of Vacation Marketing. 2001, V. 7, no. 1, pp. 19-30.

NICOLAU, J. L.; MÁS, F. J.Heckit modelling of tourist expenditure: Evidence from Spain. En:International Journal of Service Industry Management. 2005. V. 16, no. 3, pp. 271293

OPPERMANN, M.Destination threshold potential and the las of repeat visitation. En:Travel Research. 1998, V. 37, pp. 131-7.

OSTROWSKI, P.; O'BRIEN, T.; GORDON, G. Determinants of service qualityin the comercial airline industry: differences between business and leisure travelers. En: Journal of Travel and Tourism Marketing. 1994, V. 3, pp. 19-47.

REICHHELD, F. F.; SASSER, W. E. Zero defections: Quality comes to services. En:Harvard Business Review. 1990, V. 68, pp. 105-111.

RITCHIE, J. R. B.; FILIATRAULT, P.Family vacation decision-making -A replication and extension. En: Journal of Travel Research. 1980, V. 18, no. 4, pp. 3-14.

ROJAS-DE-GRACIA, M. M.; ALARCÓN-URBISTONDO, P.Toward a gender understanding of the influence of the couple on family vacation decisions. En:Tourism Management Perspectives,2016, V. 20, pp. 290-29.

ROJAS-DE-GRACIA, M. M.; ALARCÓN-URBISTONDO, P.Couple roles in subecisions on family vacations. En:Cornell Hospitality Quarterly, 2017, pp. 1-14.

TEYE, V. B.; LECLERC, D.Product and service delivery satisfaction amon North American cruise passengers. En:Tourism Management. 1998, V. 19, pp. 153-160.

TIDTICHUMRERNPORN, T.; JANASAK, S.; MUJTABA, B.G.; KHUNSONGKIET, P.; DUANGJAI, N.; BHAWANANTECHANON, N.; VONGKHAM, O.Measuring the satisfaction of domestic and international tourist toward Lanna cultural tourism products: A study of progress in Chiang Mai Walking Street activities. En:Journal of Business Studies Quarterly, 2010, V. 1, no. 3, pp. 31-52.

VAN RAAIJ, W. F.Consumer research on tourism mental and behavioral constructs. En:Annals of Tourism Research,1986, V. 13, no. 1, pp. 1-9.

VALERA GONZÁLEZ, J. A.Satisfacción/insatisfacción de los consumidores y comportamientos postconsumo derivados. En:Estudios sobre Consumo. 1992, V. 9, no. 23, pp. 65-79.

WANG, K. C.; HSIEH, A. T.; YEH, Y. C.; TSAI, C. W. Who is the decision-maker: The parents or the child in group package tours?. En:Tourism Management. 2004, V. 25, no. 2, pp. 183-194. 